\title{
Aspectos Éticos de la Protección Radiológica en Procedimientos de Radiología Dental
}

\author{
Ethical Aspects of Radiological Protection in Dental Radiology Procedures
}

\author{
Carlos Ubeda1'; Marco Aragón²; Gonzalo Aragón³ \& Diego Aragón ${ }^{4}$
}

UBEDA, C.; ARAGÓN, M.; ARAGÓN, G. \& ARAGÓN, D. Aspectos éticos de la protección radiológica en procedimientos de radiología dental. Int. J. Odontostomat., 15(3):577-582, 2021.

RESUMEN: Se sabe de los beneficios que la radiología dental ha significado para mejorar la calidad de vida de las personas en todo el mundo, sin embargo, también conocemos los riesgos asociados a su uso. Para disminuir estos riegos, diferentes organizaciones a nivel internacional han desarrollado un SISTEMA DE PROTECCIÓN RADIOLÓGICA (SRP) basado en los principios de: Justificación, optimización y limitación de dosis, los cuales a su vez han evolucionado en base al desarrollo de la ciencia, los valores éticos, y la experiencia acumulada en la práctica cotidiana de los profesionales de la protección radiológica. Al revisar la normativa que regula el uso de las radiaciones ionizantes en Chile, analizamos que no se tienen en cuenta los aspectos o valores éticos al momento de hablar y aplicar el SPR en procedimientos de radiología dental. Por todo lo anterior, el objetivo del presente trabajo de revisión narrativa fue elaborar un nuevo documento de consulta para los odontólogos, que contenga de manera resumida y con definiciones precisas, los principales aspectos a tener en cuenta a la hora de comprender como funciona el SPR y los valores éticos que lo fundamentan, ayudando de esta manera a resolver objetivamente los juicios de valor que se pueden presentar durante la solicitud, ejecución e interpretación de los procedimientos de radiología dental.

PALABRAS CLAVE: radiaciones ionizantes, protección radiológica, valores éticos, radiología dental.

\section{INTRODUCCIÓN}

El desarrollo de la radiología en las últimas décadas ha demostrado un claro beneficio a los pacientes en términos de expectativa y calidad de vida (ESR Executive Council 2009 \& European Society of Radiology, 2010). Sin embargo, se debe considerar que todo examen radiológico o imagenológico (con radiación ionizante) indicado a un paciente conlleva un riesgo, el cual es proporcional a la cantidad y calidad de radiación ionizante utilizada (International Commission on Radiological Protection, 2007; Okano \& Sur, 2010). Ante esto, se debe tener especial cuidado con sobreuso del diagnóstico por imágenes. Este sobreuso se entiende como cualquier procedimiento imagenológico en el que este claramente demostrado que el paciente no va a recibir un mayor beneficio (Hendee et al., 2010). Así, se ha observado en la prác- tica clínica, que el uso excesivo de exámenes radiológicos aumenta los costos de manera insostenible a los sistemas de salud, exponiendo innecesariamente tanto a los pacientes, como a la población en general a mayores niveles de radiación (Armao et al., 2012).

Desde 1925 la comunidad internacional se ha venido organizado, a través de la Comisión Internacional de Unidades y Medidas Radiológicas (International Commission on Radiation Units \& Measurements, 2020) la Comisión Internacional de Protección Radiológica (ICRP) (International Commission on Radiological Protection, 2020a), el Comité Científico de las Naciones Unidas sobre el Efecto de las Radiaciones Atómicas (United Nations

\footnotetext{
${ }^{1}$ PhD. Departamento de Tecnología Médica, Facultad de Ciencias de la Salud, Universidad de Tarapacá, Arica, Chile.

${ }^{2}$ MD. Ortodoncista. Clínica Arisa, Arica, Chile.

${ }^{3}$ Escuela de Medicina, Pontificia Universidad Católica de Chile, Santiago, Chile.

${ }^{4}$ Escuela de Medicina, Universidad de Valparaíso, Valparaíso, Chile.
} 
Scientific Committee on the Effects of Atomic Radiation, 2020) y el Organismo Internacional de Energía Atómica (International Atomic Energy Agency 2020) para así hacer un uso más seguro y eficiente de estas radiaciones, proponiendo para este objetivo, un sistema de protección radiológica (SPR), como marco conceptual y práctico para dichos fines (International Commission on Radiological Protection, 2007).

El actual SPR y sus principios de justificación, optimización y limitación han evolucionado en base a tres fuentes: la ciencia, los valores éticos, y la experiencia acumulada en la práctica cotidiana de los profesionales de la protección radiológica. EI SPR ha madurado sobre la necesidad de realizar juicio de valor en la interpretación de los riesgos y su equilibrio con los beneficios que estas aplicaciones conlleva (Cho et al., 2018).

Al revisar la normativa que regula el uso de las radiaciones ionizantes en Chile, analizamos que no se tienen en cuenta los aspectos o valores éticos al momento de hablar y aplicar la protección radiológica en procedimientos de radiología dental (Decreto Supremo № 133, Gobierno de Chile, 1984; Decreto Supremo $N^{\circ} 3$, Gobierno de Chile, 1985). Existen algunos artículos nacionales que dan cuenta de ciertos aspectos éticos asociados a las aplicaciones médicas de las radiaciones ionizantes, pero ninguno se vincula con el SPR, ni menos con la radiología dental (Möenne, 2017; Hevia et al., 2018; García, 2019). En el ámbito internacional, existe un incipiente interés por avanzar en estos temas, siendo ejemplo la reciente publicación № 138 de la ICRP que identificó los valores éticos asociados con el SPR para exposiciones ocupacionales, públicas y médicas, y para la protección del medio ambiente (Cho et al.). Actualmente, el grupo de trabajo $N^{\circ} 109$ de la misma ICRP está elaborando una nueva publicación que presentará los aspectos éticos en el uso de la radiación en la medicina, dirigida a la protección radiológica de los pacientes y destinada a profesionales médicos, pacientes, público y autoridades (International Commission on Radiological Protection, 2020b).

Finalmente, el presente trabajo de revisión narrativa tuvo como objetivo elaborar un nuevo documento de consulta para los odontólogos, que contenga de manera resumida y con definiciones precisas, los principales aspectos a tener en cuenta a la hora de comprender como funciona el SPR y los valores éticos que lo fundamentan, ayudando de esta manera a resolver objetivamente los juicios de valor que se pueden pre- sentar durante la solicitud, ejecución e interpretación de los procedimientos de radiología dental.

\section{DESARROLLO}

Sistema de protección radiológica. En uno de nuestros artículos anteriores, algo avanzamos sobre el SPR (Ubeda et al., 2018), sin embargo conviene decir que como se indica en los principios fundamentales de seguridad (International Atomic Energy Agency, 2007), "el objetivo fundamental de la protección radiológica es proteger a las personas y el medio ambiente contra los efectos nocivos de las radiaciones ionizantes". Este objetivo debe alcanzarse sin limitar indebidamente la operación de instalaciones o la realización de actividades que generan riesgos radiológicos. En consecuencia, el SPR tiene por objeto evaluar, gestionar y controlar la exposición a la radiación a fin de reducir, en la medida en que sea razonablemente posible, los riesgos radiológicos, comprendidos tanto los riesgos de efectos en la salud y como los riesgos para el medio ambiente.

El uso del modelo denominado "lineal sin umbral" es considerado actualmente como el mejor enfoque práctico para la gestión del riesgo radiológico para la protección radiológica a dosis bajas y tasas de dosis bajas y, es acorde con el "principio de prevención" (United Nations Educational, Scientific and Cultural Organization, 2005; International Commission on Radiological Protection, 2007).

Para cumplir sus objetivos el SPR, se fundamenta en tres principios. Estos son (Cho et al.):

a. Justificación: Cualquier decisión que altere la situación de exposición a radiación debería producir más beneficio que daño. Esto significa que toda decisión en virtud de la cual se introduzca una nueva fuente de radiación, se reduzca una exposición existente, o se reduzca el riesgo potencial de exposición, debería producir suficiente beneficio individual o social como para compensar el detrimento que causa dicha decisión. Se considera que el beneficio se aplica a individuos, a la sociedad en su conjunto y también al medio ambiente.

b. Optimización: La probabilidad de recibir exposiciones, el número de personas expuestas, y la magnitud de las dosis individuales deberían mantenerse tan bajas como sea razonablemente alcanzable, tenien- 
do en cuenta factores económicos y sociales. Esto significa que el nivel de protección debería ser el mejor, en las circunstancias prevalecientes, maximizando el margen de beneficio en relación al daño. Debería haber restricciones en las dosis o en los riesgos de los individuos asociados a una fuente en particular a fin de evitar resultados extremadamente injustos o no equitativos del procedimiento de optimización (restricciones de dosis o de riesgo y niveles de referencia).

c. Limitación: Las exposiciones individuales no deben exceder los límites de dosis recomendados. Sólo se aplica a situaciones de exposición planificada, que no sean la exposición médica de los pacientes o la exposición de biota no humana. Los límites de dosis reglamentarios son decididos por la autoridad reguladora teniendo en cuenta las recomendaciones internacionales, y se aplican a trabajadores y miembros del público en situaciones de exposición planificada.

Valores éticos. Fue en el Congreso de los Estados Unidos donde se inicia el debate en el año 1974 sobre los principios de la bioética, al crear la Comisión Nacional para la Protección de los Sujetos Humanos de Investigación Biomédica y del Comportamiento, que identificó los principios éticos básicos para regir la investigación con seres humanos en la medicina y las ciencias de la conducta. En 1979 se publica el "Informe Belmont" (The National Commission for the Protection of Human Subjects of Biomedical and Behavioral Research, 1979), donde distinguen tres principios éticos básicos, por este orden: respeto por las personas, beneficencia y justicia. Sin embargo, en 1979 Tom L. Beauchamp y James F. Childress, en su famoso libro "Principios de ética biomédica" (Beauchamp \& Childress, 1994), reformulan estos principios para ser aplicados a la ética asistencial o toma de decisiones éticas en medicina, distinguiendo los valores éticos de: no maleficencia, beneficencia, autonomía y justicia.

En el caso de las aplicaciones médicas de las radiaciones ionizantes y del sustento en término de los valores éticos que se deben considerar dentro de los principios del SPR, tenemos de acuerdo con lo propuesto por la ICRP (Cho et al., 2018):

a. Beneficencia/no-maleficencia: Si la beneficencia consiste en prevenir el daño, eliminar el daño o hacer el bien a otros, la no-maleficencia consiste en no causar daño a otros. Mientras que la no-maleficencia implica la ausencia de acción, la beneficencia incluye siempre la acción. Se distinguen dos tipos de benefi- cencia, la beneficencia positiva y la utilidad. La beneficencia positiva requiere la provisión de beneficios. La utilidad requiere un balance entre los beneficios y los daños. La beneficencia puede entenderse, de manera más general, como todo tipo de acción que tiene por finalidad el bien de otros.

b. Prudencia: Capacidad de pensar, ante ciertos acontecimientos o actividades, sobre los riesgos posibles que estos conllevan, y adecuar o modificar la conducta para no recibir o producir perjuicios innecesarios. Es la capacidad de tomar decisiones informadas y cuidadosas, consideradas sin el pleno conocimiento del alcance y las consecuencias de los actos. También es la capacidad de elegir y actuar sobre lo que está en nuestro poder hacer y no hacer. Originalmente, prudencia significaba "sabiduría práctica". Describe la cualidad de tener conocimiento, experiencia y buen juicio para tomar decisiones razonables y actuar en consecuencia.

c. Justicia: Es la distribución de ventajas y desventajas entre grupos de personas (justicia distributiva), justicia en la compensación de pérdidas (justicia restitutiva), y justicia en las normas y procedimientos en los procesos de toma de decisiones (justicia procesal). También se puede entender en "dar a cada uno lo suyo". Finalmente, puede ser interpretada como el tratamiento equitativo y apropiado a la luz de lo que es debido a una persona.

d. Dignidad: Es un atributo de la condición humana, la idea de que algo se debe a una persona porque es humana (valor inherente del ser humano por el solo hecho de serlo). Esto significa que todo individuo merece un respeto incondicional, independientemente de los atributos o circunstancias personales como la edad, el sexo, la salud, la discapacidad, la condición social, el origen étnico, la religión, etc. La autonomía personal es un corolario de la dignidad humana. Es la idea de que los individuos tienen la capacidad de actuar libremente (es decir, de tomar decisiones sin coerción y con conocimiento de causa) (Siurana Aparisi, 2010; Cho et al.).

\section{DISCUSIÓN}

Antes, durante y después de realizar un procedimiento de radiología dental, entran en juego una serie de reflexiones éticas y juicios de valor dado el uso de 
las radiaciones ionizantes. Primero, por parte del odontólogo prescriptor, que define la necesidad de someter a un haz de rayos $X$ a su paciente. En segundo lugar el paciente, que simplemente confía o cuestiona en el menor de los casos, exponerse a esta radiación ionizante. Tercero, el personalque ejecuta e informa el procedimiento, quiénes deben hacer todo lo posible para la seguridad y eficiencia en el uso de los rayos $X$, preocupándose no solo de la dosis recibida por el paciente y ellos mismos, sino también cuidar que las imágenes obtenidas tengan la calidad diagnóstica adecuada para alcanzar el objetivo propuesto. También proponemos un cuarto nivel de reflexión, donde estarían todos los profesionales a cargo de la garantía y control de calidad de los equipos generadores de rayos $\mathrm{X}$.

Dada naturaleza probabilística de los efectos estocásticos de las radiaciones ionizantes y las propiedades del modelo lineal sin umbral (International Commission on Radiological Protection, 2007), resulta extremadamente complejo establecer una distinción clara entre que dosis de radiación es "seguro" o "peligroso", lo cual, a su vez, crea dificultades a la hora de buscar una explicación del control a los riesgos de la radiación. La principal implicación del modelo lineal sin umbral, es que debe asumirse algún riesgo finito, aunque sea pequeño y que el nivel de protección establecido está basado en lo que se estima aceptable. Sin embargo, la evidencia científica junto con la experiencia acumulada, no asegura una correcta interpretación de los principios básicos del SPR, dado que las situaciones específicas que se pueden dar durante los procedimientos de radiología dental, son dinámicas y únicas, donde cada pacientes es diferente.

De acuerdo con Lochard et al. (2016), la aplicación de los principios del SPR es un desarrollo permanente de juicios de valor que se deberían basar en los valores éticos fundamentales, es decir, hacer más bien que mal, evitar los riesgos innecesarios, establecer una distribución justa de las exposiciones y tratar a las personas con respeto.

Así, el principio de justificación, se debería basar principalmente, aunque no exclusivamente, en el valor ético de beneficencia/no-maleficencia, o más bien en donde la relación entre hacer el bien pese más que hacer el mal. Aquí lo que se debe perseguir es que el beneficio para las personas y la sociedad, debería ser mayor que las desventajas asociadas en términos de riesgo de radiación. También este princi- pio se asocia al valor ético de la prudencia, ya que parte del riesgo de radiación y su análisis está asociado con el uso del modelo lineal sin umbral.

En cuanto al principio de optimización, éste utiliza las restricciones a las exposiciones individuales y los niveles de referencia para diagnóstico para reducir las desigualdades en la distribución de las exposiciones entre los grupos expuestos. Esta es la piedra angular del sistema. Por un lado, es un principio de acción que permite la aplicación práctica de la prudencia. Y por otro, permite la introducción de la equidad y la justicia en la distribución de las exposiciones entre los individuos expuestos, lo que hace referencia directa al valor ético de la justicia. En última instancia, teniendo en cuenta las circunstancias particulares en las que las personas están expuestas, así como sus preocupaciones y expectativas, el principio de optimización es respetar a dichas personas y tratarlas con dignidad.

En tercer lugar el principio de limitación exige que todas las exposiciones individuales y no médicas, no superen los límites de dosis recomendados. Al igual que el principio de optimización, se refiere directamente al valor ético de la prudencia, pero más aún a la justicia, al restringir el riesgo de manera equitativa para una determinada situación de exposición planificada y categoría de exposición.

Por otro lado, el cambio más grande que ha experimentado la medicina y la odontología durante el último siglo es el cambio de un modelo paternalista, en el que el medico sabia "Lo que era bueno" para el paciente, a un modelo en que el sujeto es autónomo en las decisiones que afectan su salud y su cuerpo. Así, basados en el principio de autonomía (no considerado en la propuesta de la ICRP), no debemos olvidar que es el paciente y su entorno, quienes pueden en todo momento aceptar o rechazar un procedimiento médico. Este principio se enlaza fuertemente con el deber de informar verazmente sobre los riesgos y beneficios de las radiaciones ionizantes (concepto del consentimiento informado (García et al., 2018), lo cual es sumamente complejo pues se trata de un terreno de incertidumbre y de falta de datos cuantitativos. Para resolver este dilema, los profesionales de la salud deben de recurrir al principio de justificación, es decir, si el procedimiento radiológico dental está justificado, entonces se le puede comunicar al paciente que los beneficios superan ampliamente los eventuales riesgos, y que la recomendación es someterse al procedimiento. 
Finalmente, se deben buscar niveles razonables de protección (principio de optimización) y niveles de exposición tolerables (principio de limitación) actuando siempre con prudencia (principio de justificación), es decir, procurando hacer más bien que mal (beneficencia/ no maleficencia), evitando la exposición innecesaria (prudencia), buscando una distribución justa de la exposición (justicia) y tratando a las personas con respeto (dignidad), sin olvidar su autonomía con respecto a acciones que afecten su cuerpo y su salud.

\section{AGRADECIMIENTOS}

El investigador Carlos Ubeda agradece el apoyo de la Dirección de Investigación de la Universidad de Tarapacá, a través del proyecto de investigación UTA Mayor № 7713-18.

UBEDA, C.; ARAGÓN, M.; ARAGÓN, G. \& ARAGÓN, D. Ethical aspects of radiological protection in dental radiology procedures. Int. J. Odontostomat., 15(3):577-582, 2021.

ABSTRACT: The benefits of dental radiology that improve the quality of life of populations worldwide have been well established. However, the risks associated with its use are also known. With these risks in mind, various international organizations have developed a Radiological Protection System (RPS) based on the principles of: Justification, optimization and dose limitation, which in turn have evolved based on science, values ethics, and the experience accumulated during the daily practice by radiation protection professionals. While reviewing the standards that regulate the use of ionizing radiation in Chile, we observed that ethical aspects or values are not taken into account when applying the RPS in dental radiology procedures. In light of these findings, the objective of this narrative review was to develop a new consultation procedure for dentists, which contains the main aspects to consider when understanding how SPR works, in a summarized precise form, as well as the supporting ethical values. Thereby helping to resolve the value judgments that may arise during the performance and interpretation of dental radiology procedures.

KEY WORDS: lonizing radiation, radiological protection, ethical values, dental radiology.

\section{REFERENCIAS BIBLIOGRÁFICAS}

Armao, D.; Semelka, R. C. \& Elias Jr., J. Radiology's ethical responsibility for healthcare reform: tempering the overutilization of medical imaging and trimming down a heavyweight. J. Magn. Reson. Imaging, 35(3):512-7, 2012.
Beauchamp, T. \& Childress, J. Principles of Biomedical Ethics. 4th ed. New York/Oxford, Oxford University Press, 1994.

Cho, K. W.; Cantone, M. C.; Kurihara-Saio, C.; Le Guen, B.; Martinez, N.; Oughton, D.; Schneider, T.; Toohey, R.; ZöLzer, F. \& Authors on behalf of ICRP. ICRP Publication 138: Ethical Foundations of the System of Radiological Protection. Ann. ICRP, 47(1):1-65, 2018.

ESR Executive Council 2009 \& European Society of Radiology. The professional and organizational future of imaging. Insights Imaging, 1(1):12-20, 2010.

García, C. La medicina defensiva en la práctica de la radiología. Rev. Chil. Radiol., 25(1):2-4, 2019.

García, C.; Moënne, K. \& Bosch, E. Consentimiento informado en radiología. Rev. Chil. Radiol., 24(2):45-7, 2018.

Gobierno de Chile. Decreto Supremo No133 (DS 133). Reglamento sobre Autorizaciones para Instalaciones Radioactivas o Equipos Generadores de Radiaciones Ionizantes, Personal que se Desempeña en Ellas, u Opere tales Equipos y Otras Actividades Afines. Santiago de Chile, Gobierno de Chile, 1984. Disponible en: https://supersalud.gob.cl/ observatorio/671/articles-8906_recurso_1.pdf

Gobierno de Chile. Decreto Supremo $N^{\circ} 3(\bar{D} S 3)$. Reglamento de Protección Radiológica de Instalaciones Radiactivas. Santiago de Chile, Gobierno de Chile, 1985. Disponible en: https://www.dt.gob.cl/legislacion/1624/articles79239_recurso_1.pdf

Hendee, W. R.; Becker, G. J.; Borgstede, J. P.; Bosma, J.; Casarella, W. J.; Erickson, B. A.; Maynard, C. D.; Thrall, J. H. \& Wallner, P. E. Addressing overutilization in medical imaging. Radiology, 257(1):240-5, 2010.

Hevia, J.; Bosch, E.; Moënne, K. \& García, C. Deber ético del radiólogo: Confidencialidad y secreto médico, respeto a la intimidad y dignidad del paciente. Rev. Chil. Radiol., 24(1):24, 2018.

International Atomic Energy Agency (IAEA). Principios fundamentales de seguridad. Nociones Fundamentales de Seguridad No. SF-1. En: Normas de Seguridad del OIEA para la Protección de las Personas y el Medio Ambiente. Viena, Organismo Internacional de Energía Atómica, 2007. Disponible en: https://www-pub.iaea.org/mtcd/publications/pdf/ pub1273_s_web.pdf

International Atomic Energy Agency (IAEA). Website, 2020. Disponible en: www.iaea.org

International Commission on Radiation Units \& Measurements (ICRU). Website, 2020. Disponible en: www.icru.org

International Commission on Radiological Protection (ICRP). Task Group 109. Ethics in Radiological Protection for Medical Diagnosis and Treatment. A Task Group under Committee 3 and Committee 4. Website, 2020b. Disponible en: http://www.icrp.org/icrp_group.asp?id=104

International Commission on Radiological Protection (ICRP). Website, 2020a. Disponible en: www.icrp.org

International Commission on Radiological Protection. The 2007 Recommendations of the International Commission on Radiological Protection. ICRP publication 103. Ann. ICRP, 37(2-4):1-332, 2007.

Lochard, J. First Thomas S. Tenforde Topical Lecture: The Ethics of Radiological Protection. Health Phys., 110(2):20110, 2016.

Moënne, K. Profesionalismo en el ejercicio de la radiología. Rev. Chil. Radiol., 23(4):193-4, 2017.

Okano, T. \& Sur, J. Radiation dose and protection in dentistry. Jpn. Dent. Sci. Rev., 46(2):112-21, 2010. 
Siurana Aparisi, J. C. Los principios de la bioética y el surgimiento de una bioética intercultural. Veritas, 22:121-57, 2010.

The National Commission for the Protection of Human Subjects of Biomedical and Behavioral Research. The Belmont Report. Office of the Secretary. Ethical Principles and Guidelines for the Protection of Human Subjects of Research. Bethesda, The Commission, 1979. Disponible en: https://www.hhs.gov/ ohrp/regulations-and-policy/belmont-report/read-the-belmontreport/index.html

Ubeda, C.; Nocetti, D. \& Aragón, M. Safety and radiological protection in dental imaging procedures. Int. J. Odontostomat., 12(3):246-51, 2018.

United Nations Educational, Scientific and Cultural Organization (UNESCO). The Precautionary Principle. Paris, United Nations Educational, Scientific and Cultural Organization, 2005.

United Nations Scientific Committee on the Effects of Atomic Radiation (UNSCEAR). Website, 2020. Disponible en: www.unscear.org
Dirección para correspondencia:

Carlos Ubeda, PhD.

Departamento de Tecnología Médica

Facultad de Ciencias de la Salud

Universidad de Tarapacá

Arica

CHILE

E-mail: carlos.ubeda.uta@gmail.com 